Abstract

\title{
The Addition of High-Load Resistance Exercises to a High-Intensity Functional Training Program Elicits Further Improvements in Body Composition in Trained Healthy Adults ${ }^{\dagger}$
}

\author{
Georgios Posnakidis 1, George Aphamis ${ }^{1}$, Christoforos D. Giannaki ${ }^{1}$, Vassilis Mougios ${ }^{2}$, \\ Panayiotis Aristotelous ${ }^{1}$ and Gregory C. Bogdanis $3, *$ \\ 1 Department of Life and Health Sciences, University of Nicosia, CY-1700 Nicosia, Cyprus \\ 2 Laboratory of Evaluation of Human Biological Performance, School of Physical Education and Sport \\ Science at Thessaloniki, Aristotle University of Thessaloniki, 54124 Thessaloniki, Greece \\ 3 School of Physical Education and Sports Science, National and Kapodistrian University of Athens, 17237 \\ Athens, Greece \\ * Correspondence: gbogdanis@phed.uoa.gr \\ + Presented at the 9th Greek Conference of Biochemistry and Physiology of Exercise, Thessaloniki, Greece, \\ 18-20 October 2019.
}

Published: 3 September 2019

\begin{abstract}
AIM: The aim of this study was to examine whether the addition of high-load resistance exercises to a high-intensity functional training (HIFT) program elicits further improvements in physical fitness-related parameters and body composition. Material \& Method: Twenty recreationally active volunteers ( 8 male, 12 female; age, $30 \pm 4$ y; body mass, $65.8 \pm 12.7 \mathrm{~kg}$; height, $167 \pm 7 \mathrm{~cm}$ ) were randomly assigned to a HIFT-control (HIFT-C, $n=10$ ) or HIFT-power group (HIFT$\mathrm{P}, n=10)$ and trained 3 times per week for 8 weeks. The HIFT-C protocol consisted of four rounds of an 8-exercise circuit (30:15 s work:rest, 2 min rest after round 2), which included clean-and-press jump box, TRX chest press, wall ball throws, burpees, repeated $10 \mathrm{~m}$ sprints, sumo squat-andupright row (at 65\% 1RM), and abdominal crunches. The HIFT-P group replaced the TRX chest press with bench chest press and the squat-and-upright row with squat at $80 \% 1 \mathrm{RM}$. Before and after training, participants underwent evaluation of body composition, cardiorespiratory fitness ( $\mathrm{VO}_{2 \mathrm{max}}$ ), vertical jump, 1RM bench press, and maximum number of abdominal crunches in $1 \mathrm{~min}$. Two-way repeated-measures ANOVA was used to analyze results. Statistical significance was set at $p<0.05$. Results: After 8 weeks the following parameters improved in both groups: $\mathrm{VO}_{2} \max (5.2$ $\pm 5.4 \%, p=0.003)$, squat jump $(10.9 \pm 9.8 \%, p<0.001)$, countermovement jump $(8.0 \pm 6.0 \%, p<0.001)$, bench press $1 \mathrm{RM}(18.6 \pm 19.6 \%, p<0.001)$, and body fat mass $(0.82 \pm 1.65 \mathrm{~kg}, p<0.001)$. However, muscle mass increased only in HIFT-P $(3.3 \pm 2.3 \%, p=0.002)$ and abdominal muscle endurance improved only in HIFT-C $(16.2 \pm 12.2 \%, p=0.002)$. Conclusions: Short-term HIFT resulted in improvements in whole-body cardiorespiratory and neuromuscular fitness and reduction of body fat. The addition of high-load resistance exercises to a HIFT training program was well tolerated and resulted in increased muscle mass.
\end{abstract}

Keywords: body composition; high-intensity functional training; strength; $\mathrm{VO}_{2}$ max 